\title{
Field Validation of Generic Type 4 Wind Turbine Models Based on IEC and WECC Guidelines
}

\author{
A. Lorenzo-Bonache, A. Honrubia-Escribano, F. Jiménez-Buendía, E. Gómez-Lázaro, Senior Member, IEEE
}

\begin{abstract}
The generic wind turbine models developed in recent years by the International Electrotechnical Commission (IEC) and the Western Electricity Coordinated Council (WECC) are intended to meet the needs of public, standard, and relatively simple (small number of parameters and computational requirements) wind turbine and wind farm models used to conduct transient stability analysis. Moreover, the full-scale converter (FSC) wind turbine technology referred to as Type 4 by IEC and WECC, is increasingly used in current power systems due to its control benefits. Hence, the development of this generic model has become a priority.

This study presents the validation of two generic Type 4 wind turbine models, which have been developed in accordance with the IEC and WECC guidelines, respectively. Field data collected from a real wind turbine located in a Spanish wind farm was used to validate both generic Type 4 wind turbine models following the IEC validation guidelines. Ten different test cases are considered, varying not only the depth and duration of the faults but also the load of the wind turbine. The parameters of the models were kept constant for all the simulation cases, aiming to evaluate the accuracy of the models when facing different voltage dips.
\end{abstract}

Index Terms-Full-scale converter, Generic model, IEC 6140027, Power system stability, Type 4, WECC, Wind turbine.

\section{INTRODUCTION}

$\mathbf{F}$ OR MANY YEARS, power system operators have had to deal with the lack of wind turbine (WT) and wind farm (WF) models adapted to their needs. Each WT model is usually provided by the manufacturer, and is defined to cover its own needs, but not to conduct transient stability analysis of large networks, as required by TSOs and DSOs (transmission and distribution system operators, respectively). Moreover, these vendor models are usually confidential [1], complex, and specifically defined for each WT configuration. These characteristics present a major problem for power system operators who have to work with a huge amount of information to perform their power system studies.

To contribute to the integration of wind energy into power systems, and aiming to solve these issues, the International Electrotechnical Commission (IEC) and the Western Electricity Coordinating Council (WECC) have developed generic, also known as standard or simplified, wind turbine and wind farm models [2]. These models are intended to be public, are defined by a limited number of parameters, are easily implemented in any simulation software, and use relatively low computational resources. These two organizations classify the

A. Lorenzo-Bonache, A. Honrubia-Escribano and E. Gómez-Lázaro are with the Renewable Energy Research Institute and DIEEAC-EDII-AB Universidad de Castilla-La Mancha, 02071 Albacete, Spain (email: alberto.lorenzo@uclm.es; andres.honrubia@uclm.es; emilio.gomez@uclm.es)

F. Jiménez-Buendía is with Siemens Gamesa Renewable Energy. Pamplona, Spain (email: fjimenez@gamesacorp.com) different topologies of WTs in four types, mainly according to the electrical generator [3]. In 2009, the IEC started to develop generic WT models, publishing the first Edition of the IEC 61400-27-1 in February 2015 [4]. This edition described the four WT model types [5], omitting the WF modeling for the 27-2, currently under development. The WECC guidelines were firstly published in 2010 [6], and included the modeling of WTs and WFs. However, as their response was unsatisfactory [7], the "Second Generation of Wind Turbine Models" [8] was released in 2014. These two organizations aim to provide generic models of WT and WF, which can emulate any vendor topology, and which can be used to perform transient stability analysis with sufficient accuracy, without the need for more complex models. In this sense, one of the most interesting fields of study is the compatibility between the generic WT models defined by the two entities. At first, both working groups developed the models in conjunction, with the assistance of a certain group of manufacturers. However, notable differences related to approaches adopted in the aim of these models have been identified. The IEC focuses on ensuring the most accurate response, while the WECC focuses on minimizing the number of parameters, as well as the simulation time. This difference in approaches has led to a number of similarities an differences between these generic models, which require further attention [9].

Under this framework, the present paper first presents the implementation of the generic Type 4 WT models defined by IEC and WECC in MATLAB/Simulink. Type 4 represents a wind turbine with full-scale converter (FSC) technology [10]. This WT configuration is now increasingly used, because it permits the possibility of not including gearbox, and due to the electrical control facilities provided by the full-scale converter [11]. The main drawback is the need for a converter sized to the WT rated power, which is more expensive than the commonly used doubly-fed induction generator (DFIG) configuration, composed of a converter rated to $25-30 \%$ of the WT rated power. In fact, the validation of a generic DFIG model was performed by the authors in [12]. Once implemented, both Type 4 models are tested in 10 different cases: 5 three-phase voltage dips when the WT is injecting its nominal power, and then 5 three-phase voltage dips with the same voltage parameters, but with the WT injecting 10$20 \%$ of its nominal power. Each pair of voltage dips is defined with different values of depth and duration. Since neither of these guidelines have developed a negative-sequence model, these models can only be tested in the case of threephase faults [13]. Subsequently, the responses of four different magnitudes (active power, active current, reactive power, and reactive current) are compared with the real responses of a 
Type 4 WT located in a Spanish wind farm (provided by the wind turbine manufacturer Siemens GAMESA). Finally, the validation method defined by the IEC is applied to the comparison of the two models with the field data. IEC 6140027-1 has defined three categories of parameters: "Type", which is specific to a WT model; "Project", which is specific to the project (e.g. the control parameters to comply with a grid code); and "Use case", which can be modified for each simulation (e.g. depending on the load of the WT). Since most of the WT model parameters are Type or Project, the authors prioritized keeping them constant for all study cases, guaranteeing that once the model is adjusted, it can be used for any simulation test case.

The scientific literature includes several works focused on the implementation of the IEC [14], [15] and the WECC [16], [17] generic Type 4 WT models. Furthermore, the validation of the WECC generic Type 4 WT model has been discussed in [18]-[20] (in fact, the WECC guidelines provide validation responses for their models [8]). However, the IEC generic Type 4 model validation has not been analyzed in depth, with only a few works being published before the IEC 61400-271 [21], [22]. Moreover, none of these includes a wide range of voltage dips or considers different operating conditions. Therefore, the present work aims to fill this gap with the following main contributions: $i$ ) Providing guidelines in the modeling of the generic Type 4 WT model proposed by IEC and WECC, ii) Testing the capability of the generic models to emulate the behavior of a real Type 4 WT facing 10 different test cases, iii) Studying the order of magnitude of the validation errors which should be considered when working with generic WT models, $i v$ ) Testing the compatibility between the models defined by the IEC and WECC when facing the same event.

Following this introduction, the paper is structured as follows: Section II presents the main characteristics and the implementation of the generic Type 4 WT model following the IEC and WECC guidelines. Section III describes the methodology followed to obtain the field measurements and the model simulations, as well as the IEC validation method. The results are then presented in Section IV. Finally, the conclusions of the paper are presented in Section V.

\section{GENERIC TYPE 4 WT MODEL}

The generic Type 4 WT model represents a full-scale converter WT. Both international organizations, the IEC and the WECC, subdivide Type 4 in two types, depending on the simulation of the mechanical oscillations. These oscillations mainly depend on the characteristics of the real WT. The real Type 4 WT used for this work was a full converter equipped with a brake chopper, which absorbs the excess power. Thus, pitch and speed are unaffected since the generator experiences no change in power generation. Consequently, the authors modeled a generic IEC Type 4A and a generic WECC Type 4B, which neglect the mechanical and aerodynamic parts, and thus no power oscillations are simulated.

The modular structure of a generic Type $4 \mathrm{WT}$ is shown in Fig. 1. Both Type 4 models include a Control System (CS), which comprises the control systems shown in Fig. 1 (Reactive

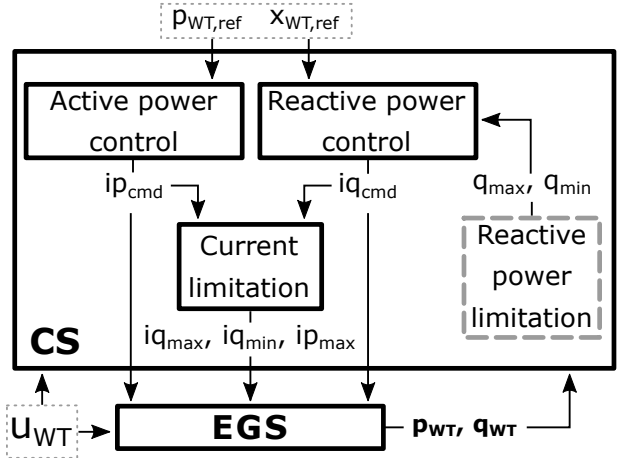

Fig. 1. Modular structure of a generic Type 4 WT.

power limitation system is only included in the IEC model). These systems provide both an active and a reactive current command signal ( $i p_{c m d}$ and $i q_{c m d}$, respectively), which are the main inputs to the Electrical Generator System (EGS), as well as the active and reactive current limits. The CS actuates following the active and reactive power references ( $p_{W T, \text { ref }}$ and $x_{W T, \text { ref }}$, respectively), which are set manually. The wind turbine terminal (WTT) voltage $\left(u_{W T}\right)$, defined as the profile of its module and angle, is an input to the CS and the EGS. Finally, the EGS provides the responses of active and reactive currents and power. In the present paper, $p_{W T, \text { ref }}$ is defined by measuring the steady-state value from the real WT. With regard to the reactive power, three different control modes can be selected: voltage in the WTT, reactive power injected, or power factor. For this work, the reactive power injected is directly controlled, the value being set in the same way as that of the active power. In this sense, this behavior is followed by the real WT during steady or dynamic state (no voltage dip operation).

Since the present paper analyzes the behavior of a real WT facing voltage dips, the transient control strategies used by the models are described as follows. Regarding reactive power transient control, the main scheme is shown in Fig. 2. F_UVRT is a variable with a value of 0 during normal operation, 1 during the fault $\left(u_{W T}<0.9 \mathrm{pu}\right)$ and 2 during a certain post-fault period. $i q_{b a s e}$ is the reactive current commanded by the normal operation controller. Hence, during steady-state operation, current $i q_{c m d}$ is commanded by the current controller (i.e. $i q_{c m d}=i q_{b a s e}$ ); during the fault it is calculated as $i q_{b a s e}$ plus a value proportional to the depth of the dip ( $T_{u s s}$ is sufficiently large to maintain the steady-state value during the fault, e.g. $100 \mathrm{~s}$ ), and after the fault, a constant value $i q \_p o s t$ is added to $i q_{\text {base }}$ for a certain time. The real WT follows this behavior, but the relationship between the dip depth and the current injected $(K q v)$ is defined as a curve, not as a constant. Furthermore, the it injects no additional reactive power during the fault $\left(i q \_\right.$post $\left.=0\right)$.

With regard to active power, none of the documents specifies any transient control systems in CS (WECC includes one in the EGS). This involves difficulties when modeling certain behaviors of a real WT. In the particular case of this paper, the real wind turbine includes a control system modifying the active power reference at a certain time after the dip, consisting 


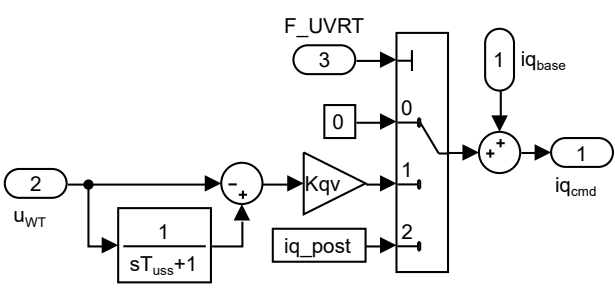

Fig. 2. Reactive power transient control system.

in recording active current just before the dip and later keeping an active current reference constant to this value during the voltage dip and $500 \mathrm{~ms}$ after. Then, the steps in active power performance shown in Section IV are due to this active power set point, as well as the limitation on active current due to reactive current priority during the dip and $500 \mathrm{~ms}$ after the voltage dip. With the aim of obtaining the most accurate response possible, the authors modeled a system similar to the one shown in Fig. 2, modifying the reference after the fault proportionally to the dip depth.

In fact, generic models should have an active control system during the faults that is proportional to voltage magnitude, since it is implemented in real WTs. This behavior is consistent with the performance required by some grid codes, such as those in the UK and Ireland, which require that active power proportional to voltage is delivered during a voltage dip. This is equivalent to freezing active current before the dip together with active power priority during the fault. The authors have considered issuing a comment to the current CDV version of the IEC 61400-27-1 (of which two of the authors are Committee members) for its consideration in Ed. 2 of the Standard.

The implementation of the Type 4 WT model following the IEC or WECC guidelines is similar because both organizations use a simple CS due to the facilities provided by full-scale converter technology. This is accentuated by the lack of mechanical model or pitch control system [23]. However, the EGS differs considerably between the two models, Fig. 3 . The WECC EGS is modeled as a filtering and saturation system with the inputs provided by CS (Fig. 3a), whereas the IEC EGS is a simplification of an actual electrical generator (Fig. 3b), further explained in [24]-[26]. Hence, the IEC EGS is a more complicated system, but with higher control possibilities.

The transient control system used in EGS also differs between the two documents. As shown in Fig. 3a, the WECC model includes the Low Voltage Active Current Management system, which multiplies the active current by a value dependent on the dip depth. Moreover, the upper limit can also be defined by LVPL (Low Voltage Power Logic). The IEC transient control system is only included in the Reference frame rotational model, and allows the voltage angle $\left(\varphi_{v}\right)$ to be filtered and/or frozen during the fault. Nevertheless, this is generally used for very severe faults (i.e. voltage under $10 \%$, because of the difficulties of measuring the angle with low voltages), and hence is not used in this work. Active and reactive ramp limiters are implemented in the real WT as well. Nevertheless, it does not use LVPL or Low Voltage Active Current Management included within WECC EGS.

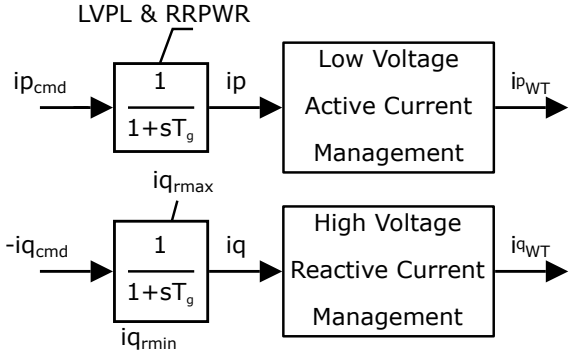

(a) WECC Type 4 EGS.

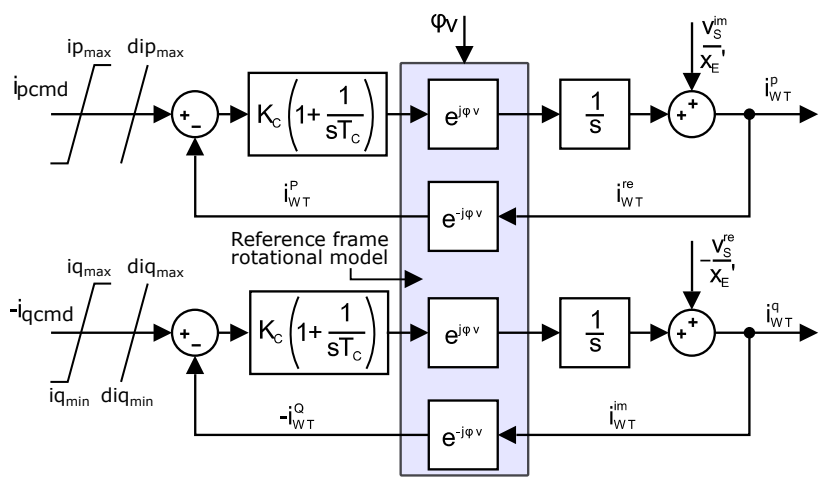

(b) IEC Type 3A EGS.

Fig. 3. Implementation of EGS for IEC and WECC.

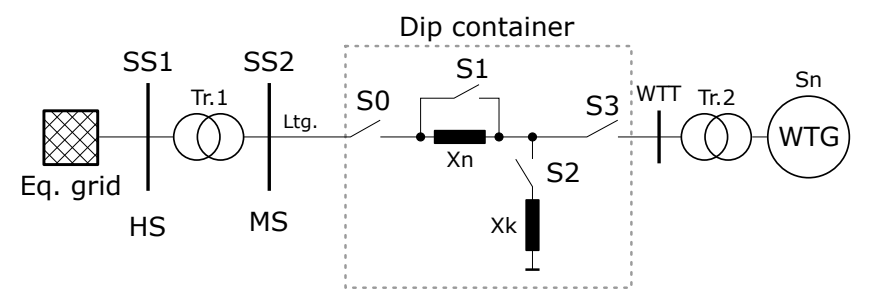

Fig. 4. Single line diagram of the FRT mobile test unit used.

\section{DESCRIPTION OF THE FIELD MEASUREMENTS AND DYNAMIC MODEL SIMULATIONS}

A fault ride-trough (FRT) mobile test unit was connected to a wind turbine located in a Spanish wind farm to perform the field tests and measurements. The use of these devices as a tool to test the FRT capability of wind turbines connected to the power system is also found in [27]-[30].

The voltage dips were forced in the medium voltage (MV) side of the transformer Tr.2 by a dip container (Fig. 4). With the aim of decreasing the current peaks, one second before applying the fault $(t=0.5 \mathrm{~s}$ in the figures shown in Section IV), a series impedance (Xn), is connected by opening a switch (S1). To generate the voltage dip, a parallel impedance (Xk) is connected by closing switch $\mathrm{S} 2$ at $t=1.5 \mathrm{~s}$. The value of this parallel impedance will affect the dip depth. Finally, one second after the fault is cleared, the series impedance is disconnected by closing switch $\mathrm{S} 1$. The connection and disconnection of S1 may cause perturbations in the behavior of the WT, as shown in Section IV, and can cause large maximum errors in some cases.

The FRT test unit is equipped with a current transformer and a voltage transformer connected to the MV side of the wind turbine. The measurement sampling rate was set to 
TABLE I

PARAMETERS OF THE TEST CASES

\begin{tabular}{|c|c|c|c|c|c|}
\hline Test & Depth (pu) & Duration (s) & $p_{W T, r e f}$ & $x_{W T, r e f}$ & Legend \\
\hline PL1 & 0.65 & 0.62 & $0.15 p u$ & \multirow{5}{*}{$\approx 0 p u$} & + \\
\hline PL2 & 0.5 & 0.92 & $0.25 p u$ & & 0 \\
\hline PL3 & 0.35 & 1.20 & $0.12 p u$ & & * \\
\hline PL4 & 0.2 & 1.70 & $0.15 p u$ & & $x$ \\
\hline PL5 & 0.1 & 2.00 & $0.13 p u$ & & $\square$ \\
\hline FL1 & 0.65 & 0.62 & \multirow{5}{*}{$\approx 1 p u$} & \multirow{5}{*}{$\approx 0 p u$} & $\diamond$ \\
\hline FL2 & 0.5 & 0.92 & & & $\triangle$ \\
\hline FL3 & 0.35 & 1.20 & & & $\triangleright$ \\
\hline FL4 & 0.2 & 1.70 & & & th \\
\hline FL5 & 0.1 & 2.00 & & & $\sum 3$ \\
\hline
\end{tabular}

$10 \mathrm{kHz}$. An anti-aliasing filter of the measurement system with a cutoff frequency equal to $4.2 \mathrm{kHz}$ was also implemented. The magnitudes obtained from the mobile test unit are: voltage in the WTT $\left(u_{W T}\right)$, active current $\left(i p_{W T}\right)$, reactive current $\left(i q_{W T}\right)$, active power $\left(P_{W T}\right)$, and reactive power $\left(Q_{W T}\right)$.

The FRT mobile unit and the test procedure previously described were used to perform ten different three-phase balanced voltage dip test cases. Specifically, voltage dip depth and duration, as well as wind turbine load conditions, were taken into account for validation purposes. Table I summarizes the parameters of all the test cases, including the voltage dip parameters, as well as the active and reactive power references $\left(p_{W T, r e f}\right.$ and $x_{W T, r e f}$, respectively). Half of the cases correspond to test cases in which the WT is operating under partial load conditions (PL), whereas in the other 5 cases the WT is operating at full load (FL). The cases for PL and FL are set in pairs (i.e. cases PL $x$ and FL $x$ share voltage dip parameters), as shown in Table I. Therefore, the experimental tests performed in this work allow for a comprehensive analysis of the Type 4 WT behavior when subjected to balanced power system faults.

The generic WT models based on both IEC and WECC guidelines, were modeled in MATLAB/Simulink. Following the IEC guidelines, the values of the parameters which define the behavior of the WT models ("Type" and "Project") are maintained constant for both models and for all the simulations. Therefore, a certain error is assumed in the responses instead of a fine adjustment for each test case. This is especially relevant for each pair of cases, in which the voltage dip has the same parameters, but the active power reference drastically changes, involving a fine adjustment of the parameters (particularly those included in the Current Limitation System).

Following the playback validation methodology defined in IEC $61400-27-1$, the measured voltage profile $\left(u_{W T}\right)$ was directly used as input to the WT generic models, thus avoiding any uncertainty related to approximated signals. The simulations were conducted with a time step of $1 \mathrm{~ms}$. Subsequently, the responses of the four signals previously mentioned, as well as the field data, went through a filter with a cut-off frequency of $15 \mathrm{~Hz}$, according to IEC 61400-27-1. Despite the validation method not being fully developed, as it will be in IEC 6140027-2, it has already been used in certain works, such as [12], [31], [32]. The validation method was applied between the IEC-Field and WECC-Field pairs, instead of comparing the responses of both generic models. Each pair of signals for each of the four magnitudes considered is subtracted, obtaining the error signal, as shown in Eq. (1).

$$
x_{\text {err }}(n)=x_{\text {sim }}(n)-x_{\text {field }}(n)
$$

Then, three different statistic indicators are obtained: the mean error (ME - Eq. (2)), the mean absolute error (MAE Eq. (3)), and the maximum error (MXE - Eq. (4)), where $n$ represents each individual sample and $N$ the total number of samples.

$$
\begin{gathered}
x_{M E}=\frac{\sum x_{e r r}(n)}{N} \\
x_{M A E}=\frac{\sum\left|x_{e r r}(n)\right|}{N} \\
x_{M X E}=\max \left(\left|x_{e r r}(n)\right|\right)
\end{gathered}
$$

Furthermore, the IEC 61400-27-1 defines different windows during the fault to calculate these errors, which are shown in Fig. 5 and depicted in Table II. Parameters $W_{\text {fault }}, W_{\text {fault } Q S}$, $W_{\text {post }}$, and $W_{\text {post } Q S}$ refer to the measurement windows, and are defined in the IEC 61400-27-1 as follows [4]:

- $W_{\text {fault }}$ : Time window covering the fault period from $t_{\text {fault }}$ to $t_{\text {clear }}$.

- $W_{\text {post }}$ : Time window covering the post-fault period from $t_{\text {clear }}$ to $t_{\text {end }}$.

- $W_{\text {faultQS }}$ : Quasi steady state part of $W_{\text {fault }}$, covering from $t_{\text {fault } Q S}$ to $t_{\text {clear }}$, where $t_{\text {fault } Q S}=t_{\text {fault }}+140 \mathrm{~ms}$

- $W_{\text {postQS }}$ : Quasi steady state part of $W_{\text {post }}$, covering from $t_{\text {clearQS }}$ to $t_{\text {end }}$, where $t_{\text {clearQS }}=t_{\text {clear }}+500 \mathrm{~ms}$

Where $t_{\text {fault }}$ is the beginning of the dip, $t_{\text {clear }}$ is the end of the dip, $t_{\text {end }}$ is the end of measurements.

$t_{\text {fault }}$ and $t_{\text {clear }}$ are followed by transient time periods which cannot be appropriately simulated by fundamental frequency models. Thus, MAE during the fault and MXE during and after the fault are calculated in these quasi steady windows. The behaviors that cannot be emulated are not considered. These misbehaviors are due to the limitations when replicating events such as the DC-component of the generator flux, the transformer inrush or the non-linear aerodynamic effects.

Therefore, each validation error is obtained for each magnitude ( $i p_{W T}, i q_{W T}, P_{W T}$ and $Q_{W T}$ ), as well as for the fault and the post-fault periods.

For the present paper, the ME was dismissed as it can take either positive or negative values, which complicates the comparison between different cases. Moreover, the MAE represents a very similar magnitude, which always takes positive values. Hence, the MAE and the MXE were considered to test the validity of the generic models in this work. 


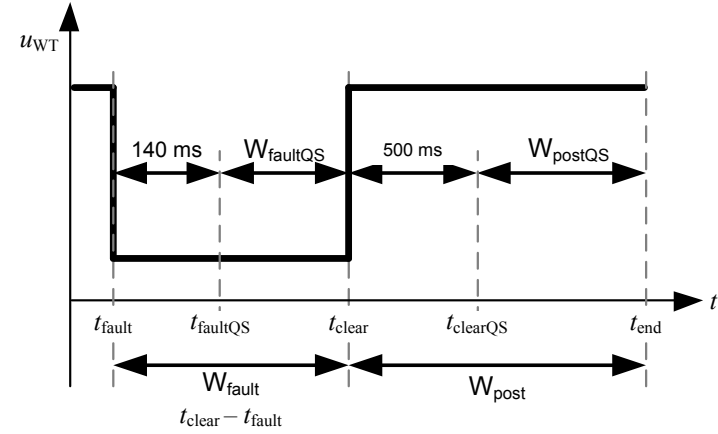

Fig. 5. Voltage dip windows.

TABLE II

WINDOWS APPLIED FOR ERROR CALCULATIONS

\begin{tabular}{c|ccc} 
Period & $x_{M E}$ & $x_{M A E}$ & $x_{M X E}$ \\
\hline Fault & $W_{\text {fault }}$ & $W_{\text {faultQS }}$ & $W_{\text {faultQS }}$ \\
Post-Fault & $W_{\text {post }}$ & $W_{\text {post }}$ & $W_{\text {postQS }}$
\end{tabular}

Regarding the simulation signals, the field measurements performed finished $1 \mathrm{~s}$ after the fault was cleared and the simulation time was set for all the test cases at $5 \mathrm{~s}$. Once the field data end, the final value is kept constant until $5 \mathrm{~s}$ is reached. However, the validation is only conducted within the period in which there are real measurement values (i.e. $W_{\text {postQS }}=0.5 \mathrm{~s}$ ).

\section{Results}

The results are presented in 3 parts: $i$ ) subsection IV-A shows the validation error distribution during the fault and the post-fault period, distinguishing between the results obtained by the IEC and WECC models; ii) subsection IV-B shows a statistical analysis in box plots, in which the aggregate errors of IEC and WECC models are compared with PL and FL test cases; iii) subsection IV-C focuses on analyzing the most relevant test case results, emphasizing those which complement the previous results.

\section{A. IEC and WECC validation error comparison}

Fig. 6 and Fig. 7 show the MAE and the MXE for all the test cases listed in Table I. The legend for these graphics is shown in the last column of Table I. For each validation error column, the left-hand symbol represents the error obtained with the IEC model, while the right-hand symbol represents the error obtained with the WECC model. For example, in Fig. 6, the MAE for active power in test case PL1 (symbol +) takes values of $0.70 \%$ and $0.80 \%$ for the IEC and WECC models, respectively. The dotted line between the validation error of both models is a visual aid, representing no further relationship. It is worth noting that both figures represent a total of 320 data.

Regarding Fig.6, the validation error obtained is very similar for the IEC and WECC generic models, except for certain cases, such as the MXE in $Q$ and $i q$ for PL4 $(\times)$. For the MAE of $Q$ and $i q$, a slight tendency is observed in which the

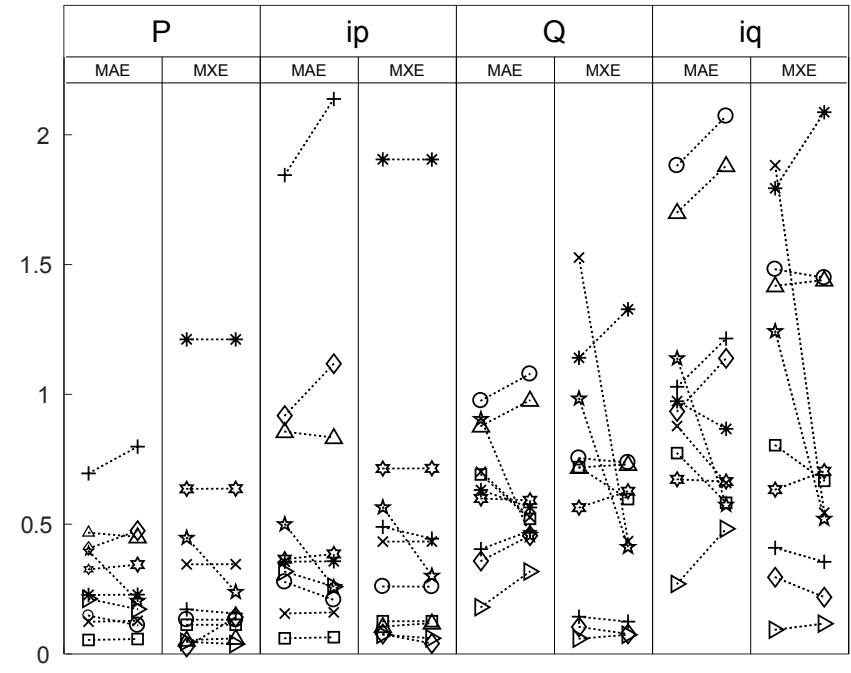

Fig. 6. Validation error distribution during fault period.

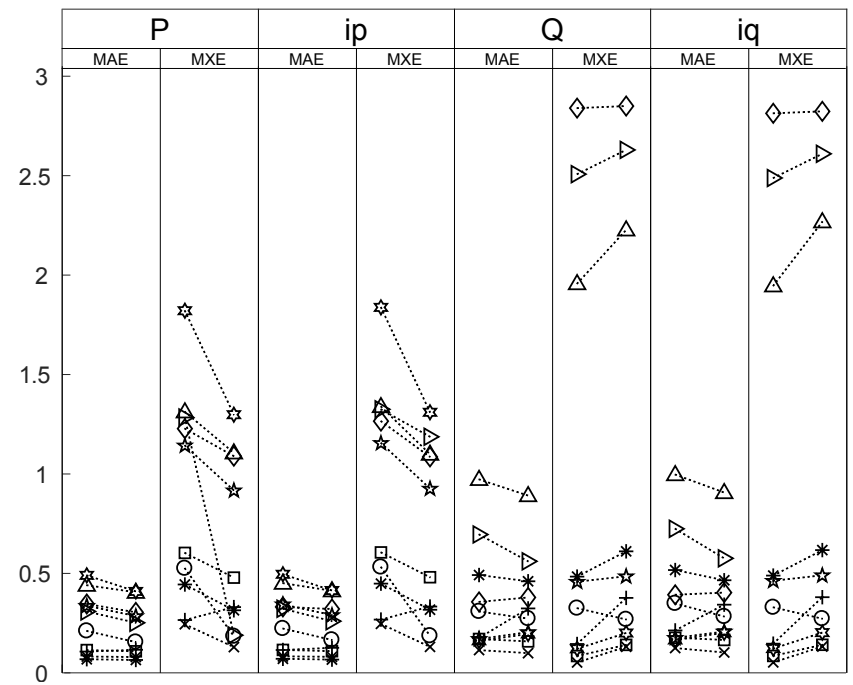

Fig. 7. Validation error distribution during post-fault period.

IEC validation error is smaller than the WECC error. During the fault period, the error between the magnitudes $P-i p$ and $Q-i q$ is related to the depth of the voltage dip, as the power magnitude is essentially the current magnitude multiplied by the voltage. Finally, it can be seen that the maximum errors during the fault period are just over $2 \%$, as will be discussed in the following subsection.

With regard to the validation errors obtained during the post-fault period, Fig. 7, a more significant trend is observed between the errors obtained for both models. For $P$ and $i p$, in most of the test cases, the MAE and MXE for the IEC generic model present higher values than the WECC model, as well as the MAE in $Q$ and $i q$. In contrast, the MXE in these last two magnitudes presents higher values for the WECC model. It is also worth noting that during the post-fault period, the MXE usually takes higher values (just under 3\% in some cases) than the MAE. This behavior will be discussed in depth in subsection IV-C. 


\section{B. Comparison between $P L$ and FL test cases}

In this subsection, the distribution of the validation errors of the generic models is analyzed using the box plots shown in Fig. 8 and Fig. 9. In each box, the $25^{\text {th }}$ and $75^{\text {th }}$ percentiles are represented by the bottom and top edges of the box respectively, while the central mark represents the median of the data. The most extreme values are represented by the extension of the whiskers, except the outliers (i.e. data which are distant from the rest), which are individually marked as ' + '. In this case, the errors from the IEC and WECC models are analyzed together, as well as the group of errors which are obtained from the set of studies at partial load (PL) or full load (FL). As an example, in Fig. 8, the box of the MAE during the fault for PL studies comprises the errors obtained in tests PL1-PL5 for WECC and IEC (i.e. each box is formed by 10 samples), and the two crosses representing outlier values correspond to the MAE for test case PL1 (+), as can be observed in Fig. 6. In this sense, the authors considered only the statistics of active and reactive power, because of the relationship between current and power magnitudes explained in the previous subsection.

For both magnitudes, $P$ and $Q$, it is observed that test cases at PL present smaller errors than FL cases, during both fault and post-fault periods. This behavior can be analyzed as follows:

- Regarding $P$ (Fig. 8), in FL test cases, the response must decrease from a value of $\sim 1 p u$ to values within the range of the depth of the fault (for example, for FL2, depth $=0.5$ and hence $P$ decreases from $1 \mathrm{pu}$ to $0.3 \mathrm{pu}$ ). In the PL test cases, the reference is much closer to the dip value (for example, for the same dip, in PL2, $P$ decreases from $0.25 \mathrm{pu}$ to $0.15 \mathrm{pu}$ ). Hence, the adjustment may be fitter for PL test cases. In some cases, as shown in the following section, even if the signal does not change at all during the fault, the error would be $<1 \%$.

- Regarding $Q$ (Fig. 9), the difference between PL and FL errors is due, not only, to similar reasons, but also because of the existence of more disturbances in the FL test cases, as shown in the following subsection.

The only set which differs from this trend is the MXE during the fault for both $P$ and $Q$, in which the PL error is higher than the FL error. This is mainly due to test PL3 (*), which presents a large perturbation during the fault for both signals, as described in the following subsection. This large error, compared to the others in the same series, unbalances its box.

\section{Representative test cases analysis}

Aiming to complement results presented in the previous subsections, this subsection analyzes the most representative responses of the ten test cases considered in this work.

First, the active and reactive power from test PL3 are shown in Fig. 10 and Fig. 11, respectively, considering the three data sources (measured in field, IEC model response and WECC model response). Both these figures show the perturbation that unbalances the MXE during the fault period observed in the

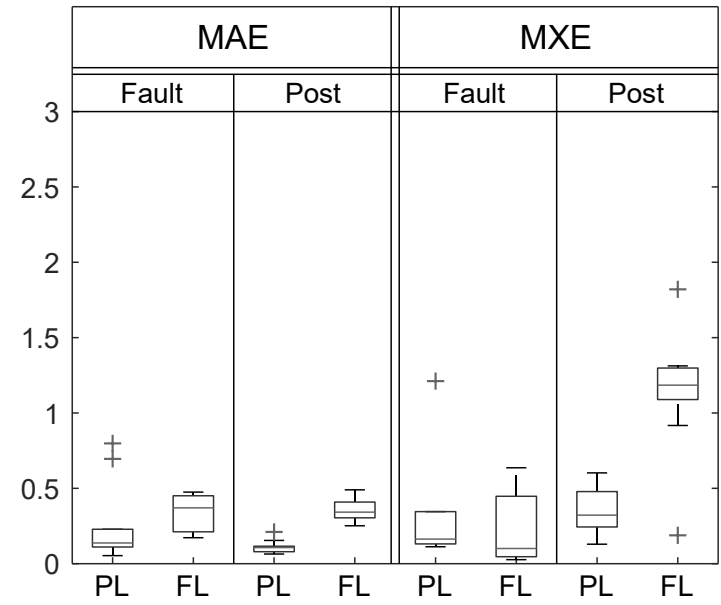

Fig. 8. Validation error analysis. Active power box plot.

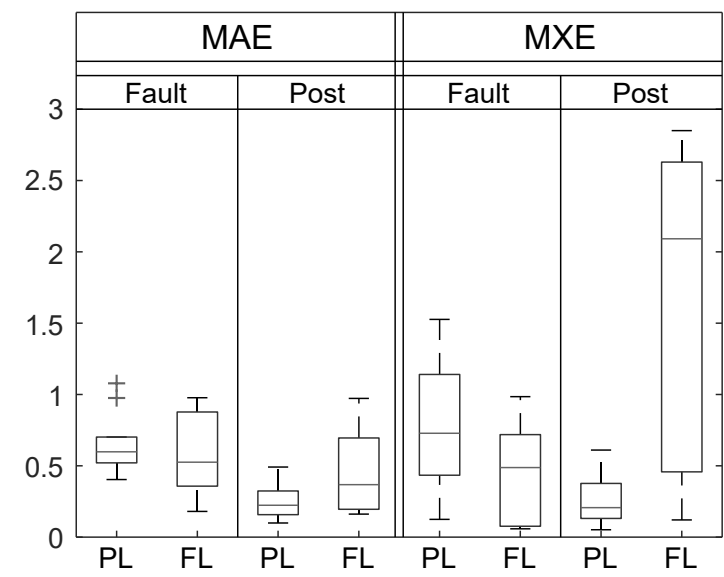

Fig. 9. Validation error analysis. Reactive power box plot.

box plots shown in the previous subsection. Specifically, in Fig. 10, it can be seen that the measured peak occurring at $2.5 \mathrm{~s}$ cannot be emulated by either of the generic models, leading to an MXE of $1.21 \%$ during the fault period for both cases. Moreover, the zoom in Fig. 11 shows that, for reactive power, the error is also $>1 \%$. Furthermore, can not only the decreasing peak not be simulated by the generic models, but also their response increases, generating even larger validation errors. This measured peak is due to a small decrease in the voltage at the WTT occurring at that time, which significantly influences the real response of the WT. Nevertheless, the active power response of the generic models is unaffected. With regard to the increase in reactive power, as shown in Fig. 2, the reactive current command during the dip is calculated adding a component that is proportional to the depth of the dip. As the voltage decrease at that moment, this additional component increases, compensating for the drop caused by the voltage perturbation.

Fig. 12 shows the active power response of test PL5, in which the depth of the dip was equal to $0.1 \mathrm{pu}$. This figure shows that for these non-severe faults with small active power references, the validation error obtained is considerably low. Even if the active power response not to vary during the fault, the mean error would be $0.5 \%$, which is negligible. This 


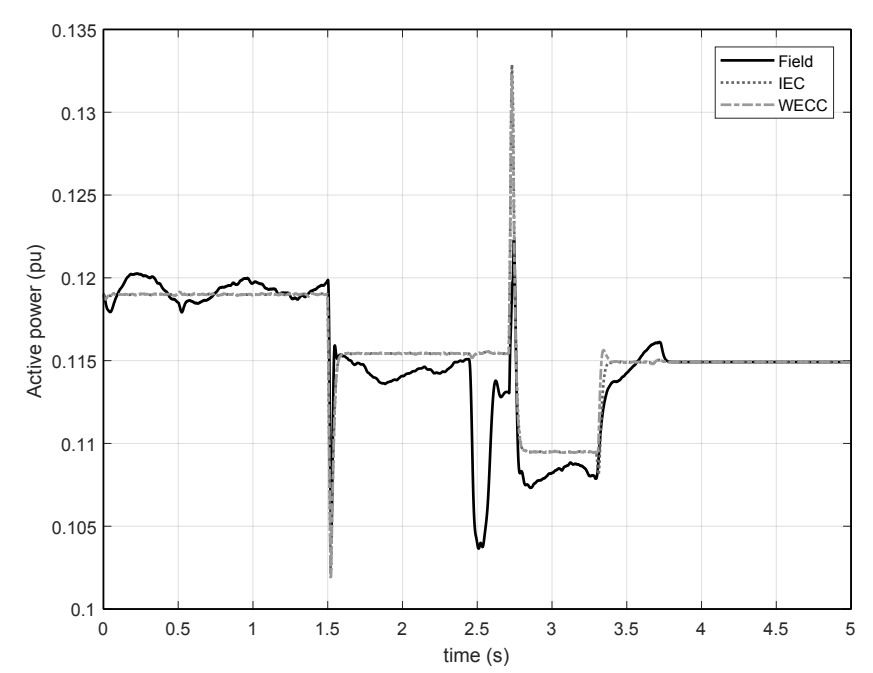

Fig. 10. Test case PL3. Active power.

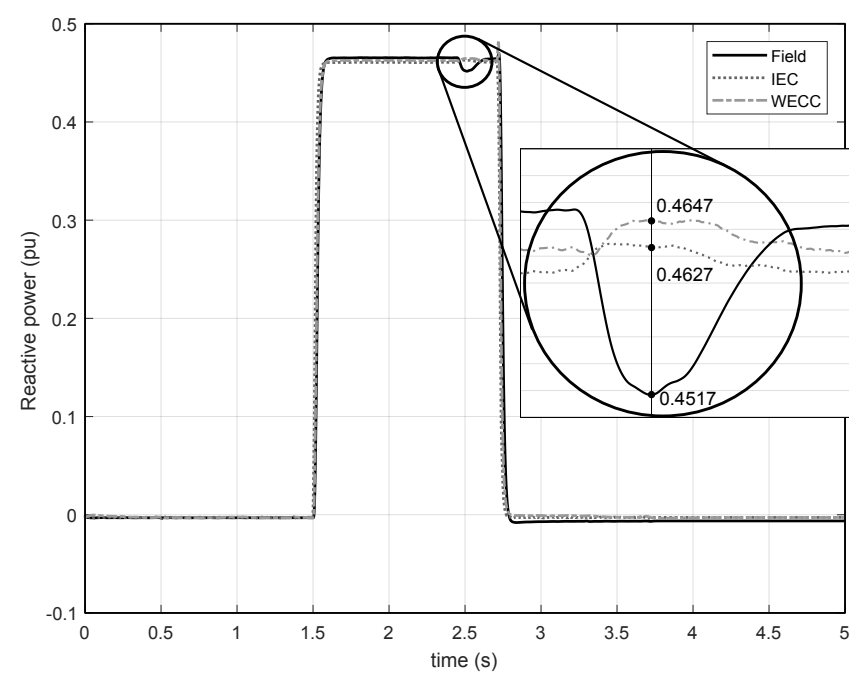

Fig. 11. Test case PL3. Reactive power.

explains the differences between the PL and FL test case boxes shown in Fig. 8.

Finally, an example of the relatively high post-fault validation errors observed under FL conditions is provided. Fig. 13 shows the active current response of the test FL3. On one hand, it can be observed that the current signal increases during the pre-fault period, due to a decrease in $u_{W T}$ in which the CS has to maintain the active power constant. On the other hand, the transient period experienced in the field by the WT, which occurs between $3.25 s<t<3.75 s$ cannot be simulated by either of the generic models, meaning MXE $>1.2 \%$. Regarding the reactive power, the zoom in Fig. 14 shows two oscillations of the WT during the post-fault period, which cannot be emulated by the generic models. A more similar response is observed for the IEC model, due to the higher control possibilities offered by its EGS. However, both models present post-fault MXE $>2.5 \%$. Furthermore, the high dispersion of MXE for FL tests shown in Fig. 9 is due to the differences between tests with and without these oscillations (i.e. the MXE is almost negligible for these cases).

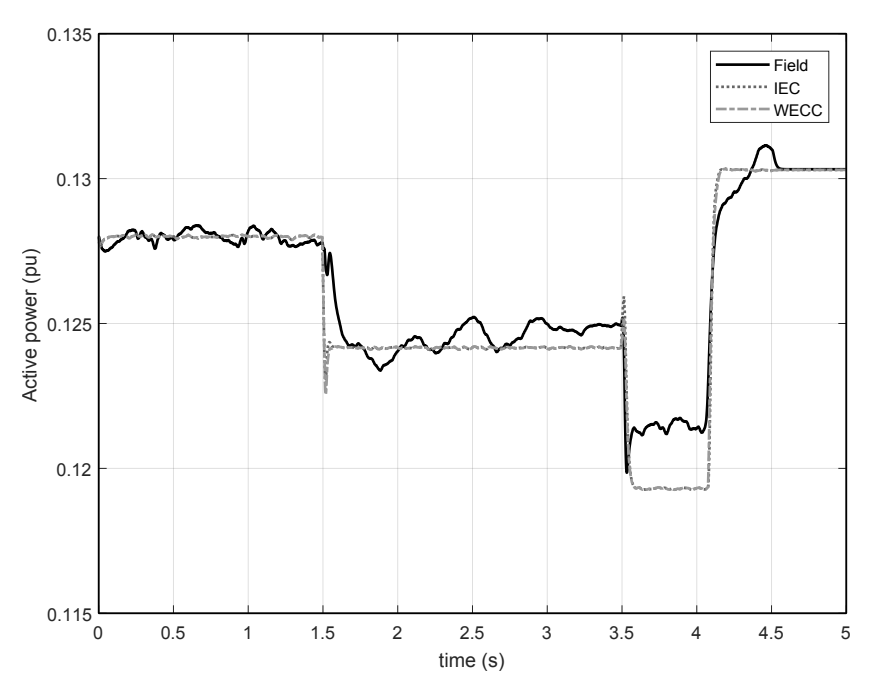

Fig. 12. Test case PL5. Active power.

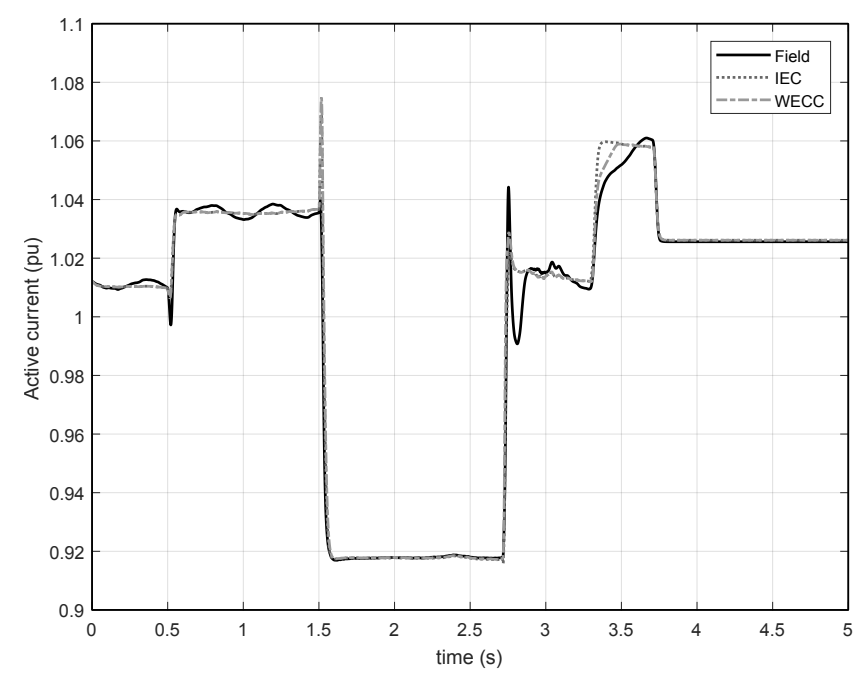

Fig. 13. Test case FL3. Active current.

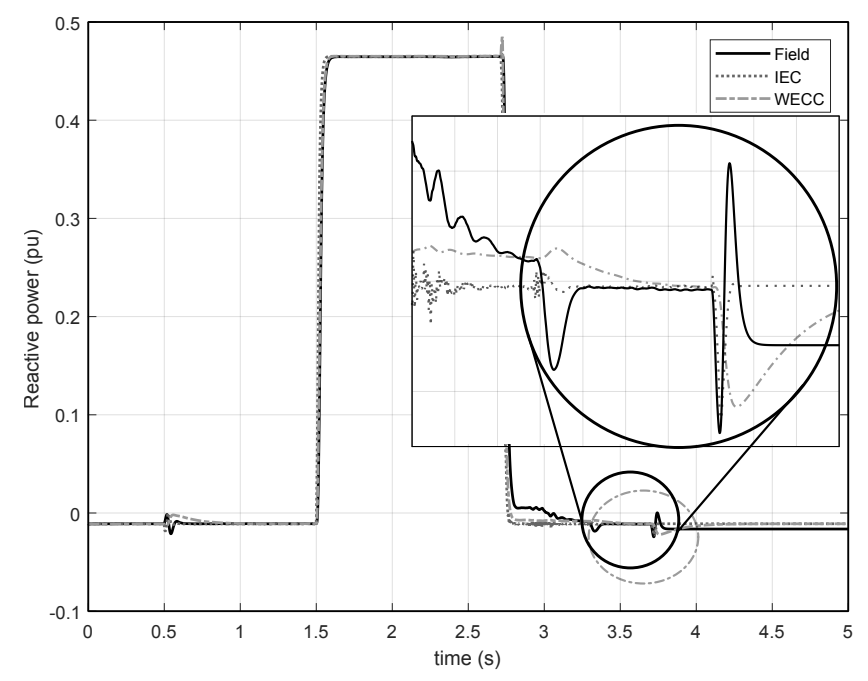

Fig. 14. Test case FL3. Reactive power. 


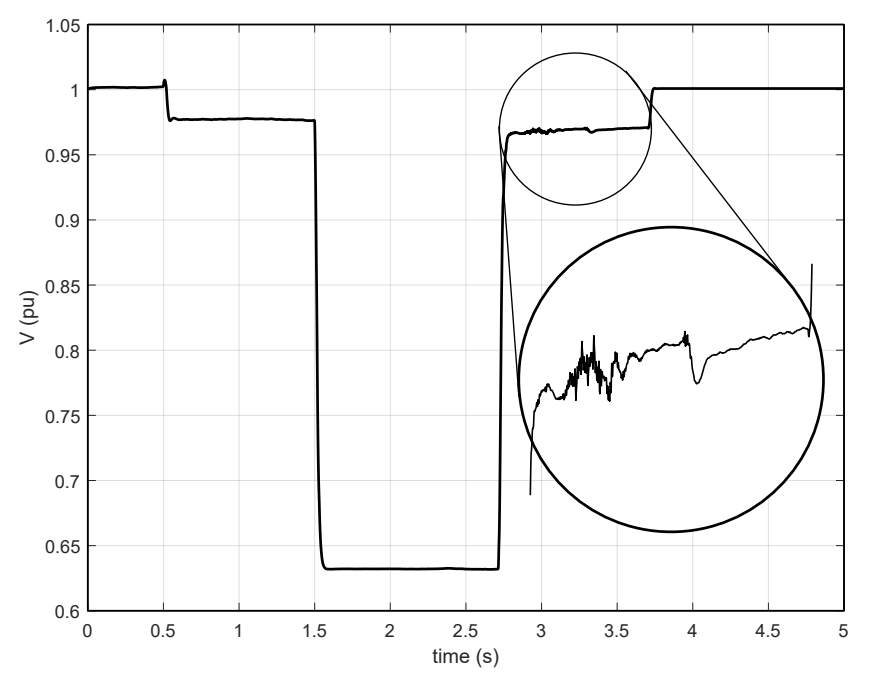

Fig. 15. Test case FL3. Voltage profile.

Both phenomena can be explained observing the voltage profile for FL3 test case, Fig. 15. When series impedance Xn is connected at $0.5 \mathrm{~s}$, the voltage decreases, generating an increase in active current, as previously explained. Furthermore, comparing Figs. 14 and 15, it can be observed that when Xn is disconnected at $3.75 \mathrm{~s}$, the perturbations causing a large MXE occur. Thus, these small fast variations of voltage cause perturbations in the responses of the WT which cannot be properly emulated by the model. Furthermore, the zoom in Fig. 15 shows the perturbations after the fault causing the irregular response in Figs. 13 and 14. It should be noted that certain real behaviors caused by small voltage perturbations cannot be reproduced by generic models.

\section{CONCLUSIONS}

The present paper validates, for the first time, the generic Type 4 WT models proposed by two international organizations: the IEC and the WECC. Both models were tested under ten different test cases, considering partial and full load conditions, as well as different voltage dip parameters. Using the validation methodology proposed in IEC 61400-27-1 (which will be fully developed in the Edition 1 of 27-2), the response of the models was compared to the response of a real Type 4 WT located in a Spanish WF. It is shown in three different ways that both generic models can accurately reproduce the general behavior of the real WT, with small validation errors for most cases $(<1 \%)$. However, some transient periods and specific responses of the WT cannot be reproduced by these generic models, leading to a number of uncommon maximum errors lower than $3 \%$ for these cases. It is also worth noting that the IEC approach, which focuses on accuracy despite the use of more parameters, allows higher control possibilities. Hence, some transient periods can be more appropriately adjusted.

Following the IEC guidelines, the authors have maintained the values of the model parameters constant for all study cases. This fact is important as all the validation errors could be reduced if the parameters were adjusted for each test. WECC guidelines do not mention this issue. However, to validate both models properly, the authors have followed the same consideration for both models.

Regarding the IEC validation method, some of the test cases show that depending on the load of the WT, the active power error may not accurately represent the variations when the dip occurs. Therefore, the validation methodology defined by IEC 61400-27-1 could include some modification considering the load of the WT, in order to obtain a more similar range of validation errors regardless of the operating condition. However, due to the recent publication of this Standard, experimental validation works, such as the present contribution, are needed to assess the capability of the validation approach.

To sum up, the generic Type 4 WT models can provide a relatively accurate response, except in particular transient periods. Nevertheless, their low computational requirements, as well as their reduced number of parameters, mean the use of these generic models is highly recommended for analysis which can accept these errors. The present paper shows a complete validation and compatibility study for Type 4 generic WT models. Thus, this work may result of particular interest to researchers, organizations, WT manufacturers or system operators considering the use of these generic models to conduct transient stability analysis. Moreover, this study might also be of interest to the IEC and WECC working groups as it contributes to testing the compatibility between both approaches, as well as the IEC validation method, which is currently under development.

\section{ACKNOWLEDGMENT}

This work was supported by the "Ministerio de Economía y Competitividad" and European Union FEDER, which supported this work under project ENE2016-78214-C2-1-R.

\section{REFERENCES}

[1] S. Seman, "Need for confidentiality. A converter manufacturer's view," 1st Wind Integration Symposium, p. 30pp, 2011.

[2] A. Honrubia Escribano, E. Gomez-Lazaro, J. Fortmann, P. Sørensen, and S. Martín-Martínez, "Generic dynamic wind turbine models for power system stability analysis: A comprehensive review," Renewable \& Sustainable Energy Reviews, vol. 81, pp. 1939 - 1952, 2018.

[3] J. Fortmann, "Generic aerodynamic model for simulation of variable speed wind turbines," in Proocedings of 9th International Workshop on Large-Scale Integration of Wind Power into Power Systems. Quebec (Canada): Energynautics GmbH, 2010.

[4] IEC 61400-27-1. Electrical simulation models - Wind turbines, International Electrotechnical Commission Std., Rev. Edition 1, February 2015.

[5] P. Sørensen, J. Fortmann, F. Jiménez Buendía, J. Bech, A. Morales, and C. Ivanov, "Final draft international standard IEC 61400-27-1. Electrical simulation models of wind turbines," 13th Wind Integration Workshop, p. $5 \mathrm{pp}, 2014$.

[6] WECC REMTF, "WECC wind power plant dynamic modeling guide," WECC, Tech. Rep., 2010.

[7] P. Pourbeik, "Proposed modifications to the WT3 and WT4 generic models," Electric Power Research Institute, Tech. Rep., 2011.

[8] WECC REMTF, "WECC second generation of wind turbines models guidelines," WECC, Tech. Rep., 2014.

[9] Ömer Göksu, P. Sørensen, A. Morales, S. Weigel, J. Fortmann, and P. Pourbeik, "Compatibility of IEC 61400-27-1 Ed 1 and WECC 2nd Generation Wind Turbine Models," 15th Wind Integration Workshop, 2016.

[10] T. T. Cristina Vázquez Hernández and A. V. Pradas, "JRC Wind Energy Status Report 2016 Edition," Market, technology and regulatory aspects of wind energy. JRC Science for policy report, 2017. 
[11] N. M. Salgado-Herrera, A. Medina-Ros, R. Tapia-Snchez, and O. AnayaLara, "Reactive power compensation through active back to back converter in type-4 wind turbine," in 2016 IEEE International Autumn Meeting on Power, Electronics and Computing (ROPEC), Nov 2016, pp. 1-6.

[12] A. Honrubia-Escribano, F. Jiménez-Buendía, E. Gomez-Lázaro, and J. Fortmann, "Field validation of a standard Type 3 wind turbine model for power system stability, according to the requirements imposed by IEC 61400-27-1," IEEE Transactions on Energy Conversion, vol. PP, no. 99 , pp. $1-1,2017$

[13] P. Pourbeik, J. J. Sánchez-Gasca, J. Senthil, J. Weber, A. Ellis, S. Williams, S. Seman, K. Bolton, N. Miller, R. J. Nelson, K. Nayebi, K. Clark, S. Tacke, and S. Lu, "Value and limitations of the positive sequence generic models of renewable energy systems," December 2015.

[14] P. Sørensen, B. Andresen, J. Fortmann, and P. Pourbeik, "Modular structure of wind turbine models in IEC 61400-27-1," IEEE Power and Energy Society General Meeting, pp. 1-5, 2013.

[15] P. Pourbeik, A. Ellis, J. Sanchez-Gasca, Y. Kazachkov, E. Muljadi, J. Senthil, and D. Davies, "Generic stability models for type 3 \& 4 wind turbine generators for WECC," IEEE Power and Energy Society General Meeting, pp. 1-5, 2013.

[16] A. Ellis, Y. Kazachkov, E. Muljadi, P. Pourbeik, and J. Sanchez-Gasca, "Description and technical specifications for generic WTG models A status report," IEEE/PES Power Systems Conference and Exposition (PSCE), pp. 1-8, 2011.

[17] P. Pourbeik, "Model user guide for generic renewable energy system models," Electric Power Research Institute, Tech. Rep., 2015.

[18] M. Behnke, A. Ellis, Y. Kazachkov, T. McCoy, E. Muljadi, W. Price, and J. Sánchez-Gasca, "Development and validation of WECC variable speed wind turbine dynamic models for grid integration studies," in AWEA's 2007 WindPower Conference, Californa, USA, Jun. 2007.

[19] M. Asmine, J. Brochu, J. Fortmann, R. Gagnon, Y. Kazachkov, C. E. Langlois, C. Larose, E. Muljadi, J. MacDowell, P. Pourbeik, S. Seman, and K. Wiens, "Model validation for wind turbine generator models," IEEE Transactions on Power Systems, vol. 26, no. 3, pp. 1769-1782, 2011.

[20] J. Bech, "Siemens experience with validation of different types of wind turbine models," IEEE Power and Energy Society General Meeting, 2014.

[21] S. Seman, J. Simolin, J. P. Matsinen, and J. Niiranen, "Validation of Type 4 wind turbine generic simulation model by full-scale test," 9th Wind Integration Workshop, p. 6pp, 2010.

[22] J. Fortmann, S. Engelhardt, J. Kretschmann, M. Janben, T. Neumann, and I. Erlich, "Generic simulation model for DFIG and full size converter based wind turbines," 9th Wind Integration Workshop, p. 8pp, 2010.

[23] A. Lorenzo-Bonache, A. Honrubia-Escribano, F. Jiménez-Buendía, A. Molina-García, and E. Gómez-Lázaro, "Generic Type 3 wind turbine model based on IEC 61400-27-1: Parameter analysis and transient response under voltage dips," Energies, vol. 10, no. 9, p. 23, 2017.

[24] C. Subramanian, D. Casadei, A. Tani, P. Srensen, F. Blaabjerg, and P. McKeever, "Implementation of electrical simulation model for IEC Standard Type-3A generator," in 2013 European Modelling Symposium, Nov 2013, pp. 426-431.

[25] J. Fortmann, "Modeling of wind turbines with doubly fed generator system," Ph.D. dissertation, Department for Electrical Power Systems, University of Duisburg-Essen, 2014.

[26] A. Lorenzo-Bonache, R. Villena-Ruiz, A.Honrubia-Escribano, and E. Gómez-Lázaro, "Comparison of a standard type 3B WT model with a commercial build-in model," IEEE International Electric Machines \& Drives Conference, 2017.

[27] F. Jiménez Buendía and B. Barrasa Gordo, "Generic simplified simulation model for DFIG with active crowbar," 11th Wind Integration Workshop, p. 6pp, 2012.

[28] F. Jiménez, E. Gómez-Lázaro, J. A. Fuentes, A. Molina-García, and A. Vigueras-Rodríguez, "Validation of a DFIG wind turbine model submitted to two-phase voltage dips following the spanish grid code," Renewable Energy, vol. 57, pp. 27-34, 2013.

[29] F. Jimenez, E. Gómez-Lázaro, J. A. Fuentes, A. Molina-García, and A. Vigueras-Rodríguez, "Validation of a double fed induction generator wind turbine model and wind farm verification following the spanish grid code," Wind Energy, vol. 15, no. 4, pp. 645-659, 2012.

[30] H. Zhao, Q. Wu, I. Margaris, J. Bech, and a. A. B. Sørensen, P. E., "Implementation and validation of IEC generic Type 1A wind turbine generator model," International Transactions on Electrical Energy Systems, vol. 25, no. 9, pp. 1804-1813, 2014.

[31] A. Honrubia-Escribano, F. Jiménez-Buendía, E. Gómez-Lázaro, and J. Fortmann, "Validation of generic models for variable speed operation wind turbines following the recent guidelines issued by IEC 61400-27,' Energies, vol. 9, 2016.

[32] M. Meuser and M. Brennecke, "Analysis and comparison of national and international validation methods to assess the quality of DG simulation models," in International ETG Congress 2015; Die Energiewende Blueprints for the new energy age, Nov 2015, pp. 1-7.

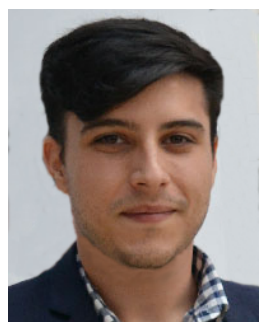

Alberto Lorenzo Bonache received the degree in electrical engineering (2015) and the master degree in industrial engineering (2017) from Universidad de Castilla-La Mancha (UCLM), Albacete, Spain. $\mathrm{He}$ is currently a Ph.D. student in Science and Technologies applied to Industrial Engineering.

Since 2015 he has been working as a researcher in the Renewable Energy Research Institute. His main research interest is the modeling of wind turbines and wind farms for the integration of wind energy into power systems and applications of real time simulation for renewable energies.

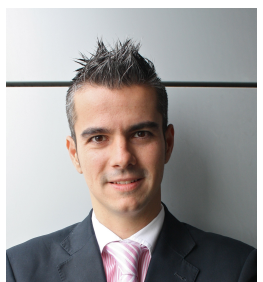

Andrés Honrubia Escribano received the degree in electrical engineering from the Polytechnic University of Madrid (UPM), Madrid, Spain, in 2008 and the Ph.D. degree in Renewable Energy from the Polytechnic University of Cartagena, Cartagena, Spain, in 2012.

Since 2008 he has worked for several research entities, publishing more than 50 articles in journals, books and specialized conferences, collaborating in more than $30 \mathrm{R} \& \mathrm{D}$ projects. Currently he is a Associate Professor in the Department of Electrical, Electronics, and Control Engineering, Castilla La Mancha University, Albacete, Spain. His main research interest is the integration of wind energy into power systems.

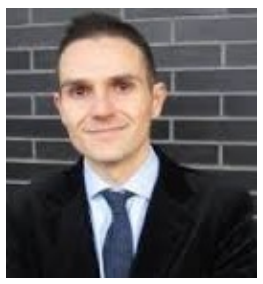

Francisco Jiménez Buendía received the M.Sc. degree in industrial engineering and the Ph.D. degree in electrical engineering from the Technical University of Cartagena, Cartagena, Spain, in 2001 and 2008, respectively.

Since 2003 he has been with GAMESA in grid integration and modeling projects. He is currently Chief Electrical Engineer for G5x product platform at SIEMENS GAMESA in Pamplona, Spain. He is a member IEC TC88 WG27. His research interests include grid integration of wind turbines into power systems, modeling of wind turbines for transient stability and development of wind power plants controllers.

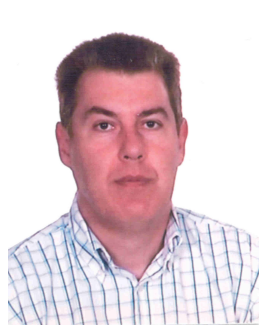

Emilio Gómez Lázaro (SM10) received the M.Sc. and $\mathrm{Ph.D}$. degrees in electrical engineering from the Universidad Politcnica de Valencia, Valencia, Spain, in 1995 and 2000, respectively.

$\mathrm{He}$ is currently a Professor in the Department of Electrical, Electronics, and Control Engineering, Castilla La Mancha University, Albacete, Spain, where he is also the Director of the Renewable Energy Research Institute. His research interests include modeling of wind turbines and wind farms, grid codes, power system integration studies, steadystate and dynamic analysis, and O\&M of renewable energy power plants. 